\title{
Single-Pass Bistatic SAR Interferometry Using Fixed-Receiver Configurations: Theory and Experimental Validation
}

\author{
Sergi Duque, Student Member, IEEE, Paco López-Dekker, Member, IEEE, and Jordi J. Mallorqui, Member, IEEE
}

\begin{abstract}
In this paper, bistatic interferometry using fixedreceiver configurations is addressed both theoretically and experimentally. The analytical expressions for interferometric phase and height sensitivity are derived, and a full interferometric processing chain for digital elevation model (DEM) generation is presented. The derived expressions are general, and they can be applied to two possible acquisition geometries: backscattering and forward scattering. The theoretical developments are complemented with experimental results done with the bistatic receiver Synthetic Aperture radar Bistatic Receiver for INterferometric Applications. The obtained DEMs are compared with a DEM from the Shuttle Radar Topography Mission and a digital terrain model from the Institut Cartografic de Catalunya. The comparison allows one to validate the results and demonstrate to which particular features of the scene that the bistatic radar is sensitive.
\end{abstract}

Index Terms-Bistatic configurations, interferometry, synthetic aperture radar (SAR).

\section{INTRODUCTION}

$\mathbf{S}$ YNTHETIC aperture radar (SAR) has been, for the last few decades, a very useful technique for Earth observation, being able to deliver images of large terrain areas independently of weather conditions. With a steady supply of data available, a number of SAR-derived techniques have been developed, such as SAR interferometry (InSAR) [1]-[4], differential InSAR (DInSAR) [5]-[7], polarimetric SAR (Pol-SAR) [8], and polarimetric InSAR [9]. Nowadays, most of these techniques have reached maturity in the monostatic case. However, bistatic and multistatic configurations are opening new lines of research. These configurations result in novel observation geometries, in some cases revealing different scattering mechanisms, and will therefore require a brand new formulation as in the case of Pol-SAR [10]. A discussion of different multistatic missions

Manuscript received April 3, 2009; revised September 15, 2009. Date of publication March 25, 2010; date of current version May 19, 2010. This work was supported in part by the Spanish Ministerio de Ciencia y Tecnologia and the European Union FEDER funds under Projects TEC2008-06764-C02-01 and TEC2007-65690/TCM, by the Spanish Ramon y Cajal program, and by the Catalan Commission for Research (CIRIT). The work of S. Duque was supported by the Spanish MEC under Grant AP2005-1484.

S. Duque and J. J. Mallorqui are with the Remote Sensing Laboratory, Universitat Politècnica de Catalunya, 08034 Barcelona, Spain (e-mail: sergi. duque@tsc.upc.edu; mallorqui@tsc.upc.edu).

P. López-Dekker is with the Microwaves and Radar Institute, German Aerospace Center (DLR), 82230 Oberpfaffenhofen, Germany (e-mail: Francisco.Dekker@dlr.de).

Color versions of one or more of the figures in this paper are available online at http://ieeexplore.iee.org.

Digital Object Identifier 10.1109/TGRS.2010.2041063 and experiments can be found in [11]-[15]. In most of these missions, however, the bistatic angle is small, so that they can be considered as quasi-monostatic from a geometrical point of view. If the receiver and transmitter follow independent trajectories and they look at the scene from different points of view, truly bistatic geometries appear.

An important issue in any multistatic system is the synchronization between the transmitter and the receivers. This problem can be solved with dedicated synchronization links, like in TanDEM-X [16]. In the case of noncooperative systems, like those using sources of opportunity, synchronization can be recovered using a dedicated channel to acquire a clean line-ofsight signal [17].

The Remote Sensing Laboratory of the Universitat Politècnica de Catalunya (UPC) is investigating bistatic configurations that use ground-based fixed receivers and orbital sensors as transmitters of opportunity. In this context, a first C-band receiver named SAR Bistatic Receiver for INterferometric Applications (SABRINA) [18], [19] has been implemented and successfully tested using the SAR systems carried by the European Remote Sensing satellite 2 (ERS-2) and ENVISAT satellites of the European Space Agency as transmitters. SABRINA is placed at locations with a good view of the scene, like the top of a hill or the roof of a tall building.

Aside from solving the technological challenges derived from working with noncooperative transmitters, the main goal of this research line at UPC is the study and development of applications based on bistatic systems. Similar to the monostatic case, interferometric applications, and particularly the differential ones, appear as a good niche. The DInSAR monitoring of urban areas affected by subsidence phenomena can benefit from the particularities of bistatic systems. For instance, in some cases, a bistatic system may be able to generate good quality data where the performance of a typical orbital monostatic geometry is severely worsened by foreshortening, which degrades the ground-range resolution, or layover effects. Moreover, a multistatic system can simultaneously observe a scene from different points of view using a single transmitter. Several receivers can be placed at different locations, and it should be possible to extract the $3-\mathrm{D}$ vector of movement by applying DInSAR techniques. The cost of deploying dedicated receivers is by far lower than having a dedicated monostatic system with similar capabilities.

This paper presents the first steps toward bistatic interferometric applications. These first steps are focused on the 


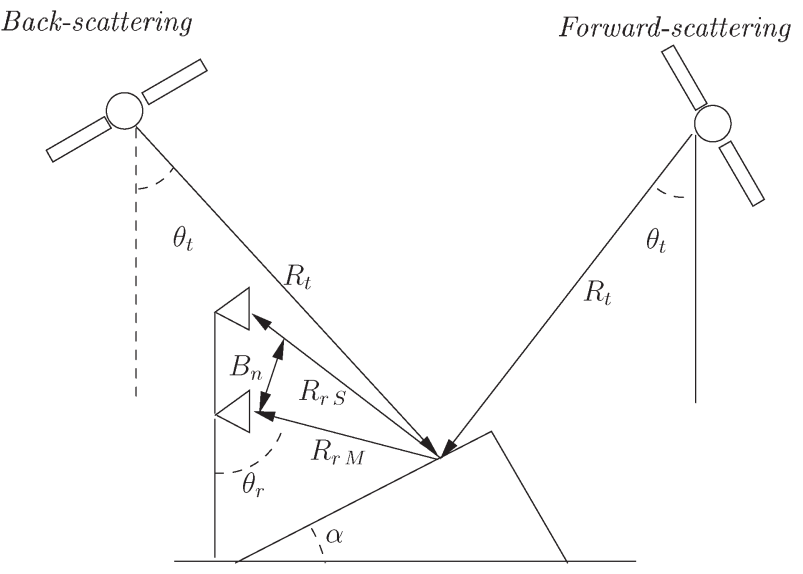

Fig. 1. Backscattering and forward-scattering bistatic SAR geometries for interferometric data acquisition.

obtention of digital elevation models (DEMs) using single-pass interferometric acquisitions. General expressions are derived, which can be applied to two different acquisition geometries: backscattering and forward scattering. The theoretical developments are complemented by the results obtained with SABRINA using ERS and ENVISAT. This paper is structured as follows: In Section II, the particular bistatic geometry with fixed receivers is discussed, as well as the expressions for range and azimuth bistatic resolutions. Section III describes SABRINA, which is the acquisition system. The particularities of the bistatic interferometric chain are highlighted in Section IV. Section V presents the bistatic DEMs and their comparison with a Shuttle Radar Topography Mission (SRTM) DEM and a digital terrain model (DTM) from the Institut Cartografic de Catalunya (ICC). Finally, Section VI presents the main conclusions of this paper.

\section{INTERFEROMETRIC GEOMETRY}

\section{A. Geometry Description}

This section analyzes a particular bistatic configuration that uses an orbital sensor as the transmitter of opportunity with a ground-based fixed receiver. Two complementary geometries are discussed: backscattering and forward scattering. In the backscattering case, the transmitter and receiver are located at the same side of the imaged area, whereas in the forwardscattering geometry, the imaged area is between the transmitter and the receiver. It has to be pointed out that, strictly speaking, pure backscattering only happens if the transmitter, receiver, and target area are aligned. Fig. 1 shows the two geometric configurations, where $\theta_{t}$ and $\theta_{r}$ are the incidence angles for the transmitter and receiver, respectively; $\alpha$ is the local terrain slope; $B_{n}$ is the perpendicular baseline from master receiver to slave receiver; $R_{r M}$ and $R_{r S}$ are the distances from a target to the master and slave receivers, respectively; and $R_{t}$ is the transmitter-target range.

\section{B. Range Resolution}

In a monostatic geometry, isorange surfaces are the spheres centered at the position of the transmitter. In a bistatic geometry, these isorange surfaces become the locus of points where the sum of distances to the transmitter and to the receiver is a constant. Thus, they are ellipsoids with the transmitter and receiver as foci. Taking this into account and considering that the terrain has local slopes $\alpha_{r}$ with respect the receiver and $\alpha_{t}$ respect the transmitter, the ground-range resolution is

$$
\left.\Delta r_{g}\right|_{\mathrm{bi}}=\frac{c}{\Delta f_{c} \cdot\left(\sin \left(\theta_{t}-\alpha_{t}\right)+\sin \left(\theta_{r}-\alpha_{r}\right)\right)}
$$

where $\Delta f_{c}$ is the bandwidth of the transmitted chirp. For our particular case, where the receiver illuminates a small scene and the transmitter is very far away, the local slopes with respect to the transmitter and the receiver are very similar $\left(\alpha_{t} \approx \alpha_{r}=\alpha\right)$. It can be approximated that the transmitter, receiver, and their respective range vectors to the scene are on the same plane, as shown in Fig. 1. Note that, in a forward-scattering geometry, $\theta_{t}$ and $\theta_{r}$ have opposite signs [19]. In the backscattering case, if both angles are equal and taking into account that $\alpha_{t} \approx \alpha_{r}=\alpha$, (1) reduces to the standard monostatic ground-range resolution expression

$$
\left.\Delta r_{g}\right|_{\text {mono }}=\frac{c}{2 \Delta f_{c} \cdot \sin (\theta-\alpha)}
$$

Fig. 2 shows a comparison of the ground-range resolution for bistatic (a) backscattering, (b) forward-scattering, and (c) monostatic geometries and assuming the $16-\mathrm{MHz}$ pulse bandwidth transmitted by ERS-2 and ENVISAT.

The incidence angle for the orbital transmitter can be considered constant along the swath (in this case, $\theta_{t}=23^{\circ}$ ) as the satellite is far away and the area of interest is relatively small. For the receiver, an incidence angle of $\theta_{r}=80^{\circ}$ has been considered. In all cases, the resolution has been plotted for angles for which there is no shadowing. Shadow regions due to the transmitter and receiver geometries are indicated. It can be observed that the monostatic geometry is less affected by the shadowing than the bistatic one. In particular, Fig. 2(b) shows the double-shadow effect present in the forward-scattering case. Observing Fig. 2(a) and (c) and taking into account (1), it can be observed that, for the regions without shadowing or layover, the backscattering bistatic range resolution is better than the monostatic while the forward-scattering resolution is worse. Still, there is a margin of incidence angles for which this forward-scattering resolution is good enough for the system to remain useful. In general, there is a total loss of resolution when the specular condition is met

$$
\left(\theta_{t}-\alpha\right)=-\left(\theta_{r}-\alpha\right)
$$

The main limitation in this example is the shadowing due to the grazing look angle of the receiver. If the receiver was set on an aircraft or if it was placed on a hill looking down to the scene, the looking angle would be better in terms of shadowing. It is also true that the shadowing region will be reduced if a set of receivers with complementary illuminated areas was 


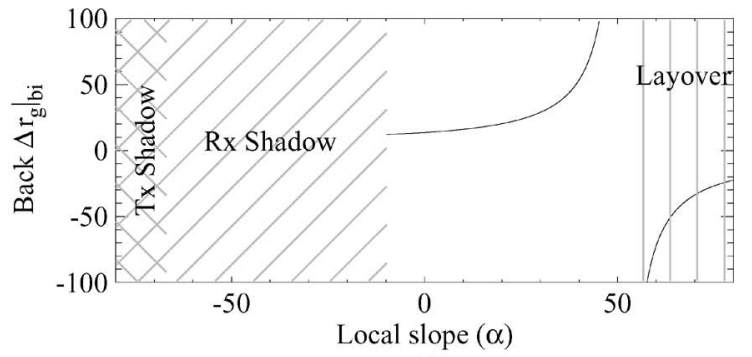

(a)

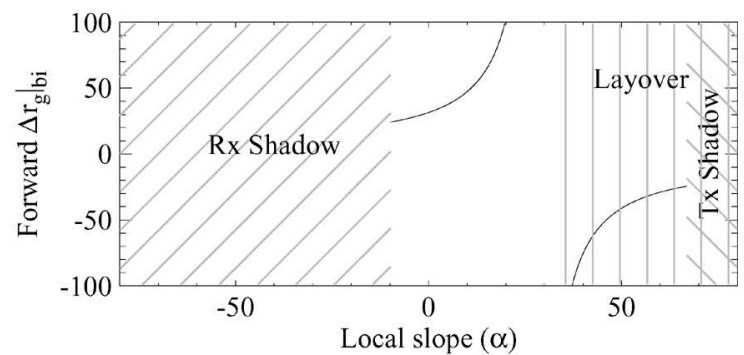

(b)

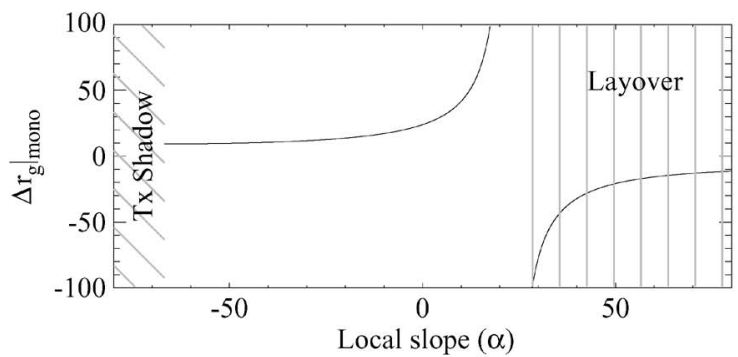

(c)

Fig. 2. Ground-range resolution in (a) backscattering, (b) forward-scattering, and (c) monostatic geometries, taking into account a receiver incident angle of $\theta_{r}=80^{\circ}$. The layover and the shadow for transmitter and receiver have been emphasized with different patterns. (a) Backscattering ground-range resolution. (b) Forward-scattering ground-range resolution. (c) Monostatic ground-range resolution.

used. It has to be noticed that the negative resolutions in Fig. 2 correspond to layover areas.

\section{Azimuth Resolution}

As it was shown in [19], the relationship between the monostatic and bistatic azimuth resolutions can be approximated by

$$
\left.\Delta r_{a}\right|_{\mathrm{bi}} \approx \frac{L_{a}}{\sqrt{2}}=\left.\sqrt{2} \cdot \Delta q r_{a}\right|_{\text {mono }}
$$

where $L_{a}$ is the azimuth effective length of the transmitting antenna. There are two phenomena that affect the azimuth bistatic resolution in comparison with the monostatic one. The first one is the loss of resolution due to the one-way propagation path of the bistatic case that reduces the effective Doppler bandwidth by a factor of two. The second is the wider one-way antenna beamwidth with respect to the two-way one that increases the integration time and, thus, the resolution approximately by a factor of $\sqrt{2}$. The combination of both leads to an effective loss of resolution roughly by a factor of $\sqrt{2}$ in comparison with the monostatic case.

\section{Interferometric Phase}

The interferometric phase for a point $P$ with respect to a reference height is

$$
\Psi_{P}=\frac{2 \pi}{\lambda} \cdot H_{P} \cdot\left(\frac{B_{n}}{R_{r M} \cdot \sin \left(\theta_{r}\right)}\right)
$$

where $H_{P}$ is the height of the point. Notice that the phase is only dependent on the one-way path from the scene to the receiver. $R_{t}$ is irrelevant as it is common to both images. As the scene is usually close to the receiver, the geometric parameters $\left(B_{n}, R_{r M}\right.$, and $\left.\theta_{r}\right)$ are expected to suffer strong variations across the swath.

\section{ACQUisition System DESCRIPTION}

The current receiver system, SABRINA, consists of a twochannel C-band front-end, where the signal is amplified and downconverted to an intermediate frequency (IF), and a highspeed digitizer that samples this IF signal. The absence of a dedicated link between the transmitter and the receiver local oscillators results in the necessity of using a direct signal for pulse repetition frequency (PRF) recovery and phase synchronization [17]. This direct signal can be obtained either from a dedicated channel, with an antenna pointing directly to the satellite, or from the direct signal recorded through the sidelobes of the receiver antenna. The former is the best option, but the latter can be used in the case of having a reduced number of digitization channels. This was the case for the interferometric experiments.

The acquisition time has to be synchronized with the satellite overpass. The acquisition window is centered in the predicted zero Doppler time. The window length has to take into account the transmitter illumination time of the region of interest and some extra margin to cope with clock synchronization and predicted orbit inaccuracies, or predicted Doppler centroid error. Thus, a conservative strategy has been adopted, consisting of sampling an acquisition window continuously within the limits of the available memory, which is roughly $8 \mathrm{~s}$.

Fig. 3(a) shows a photograph of SABRINA during an experiment at UPC, while Fig. 3(b) shows a simplified block diagram of the receiver system. For a detailed discussion of SABRINA, the reader is referred to [19].

\section{INTERFEROMETRIC PROCESSING}

\section{A. Image Focusing}

The bistatic Single-Look Complex (SLC) images can be generated using a wide range of bistatic SAR processing algorithms [12], [20]-[25]. In this paper, for simplicity, a bistatic backprojection algorithm has been used. Although this method has a high computational cost, it is appropriate in this case due to the small size of the observed scene, which is limited by the field of view of the receiver. An important consideration regarding the processing of the bistatic raw data is that the phase history is no longer azimuth invariant. The phase history of a target depends only of the variation of its relative distance with the transmitter while being illuminated. Thus, since the receiver is fixed and the bistatic distance is the sum of the distances to the target of the 


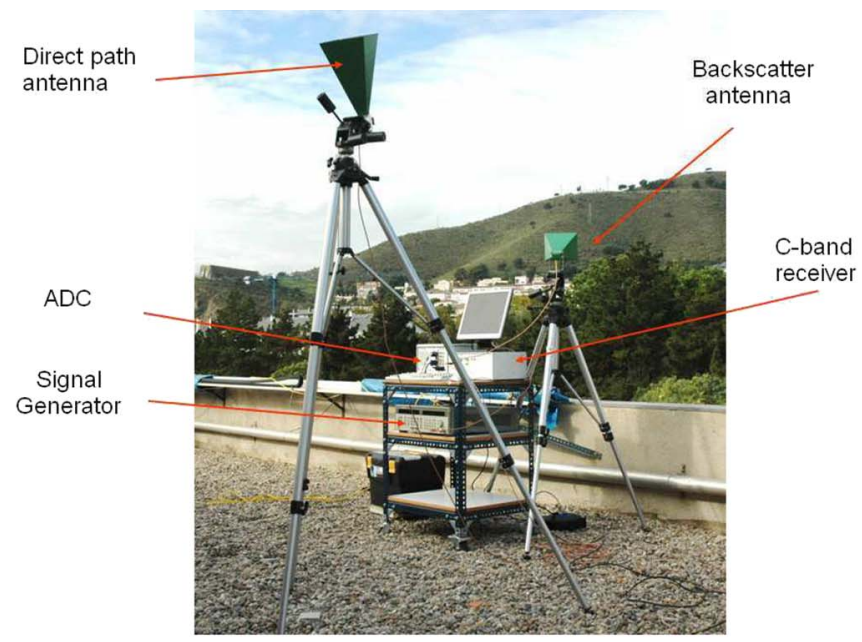

(a)

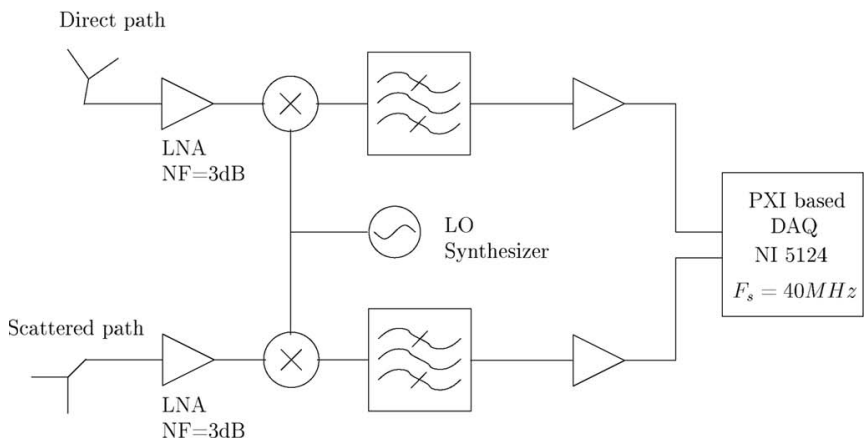

(b)

Fig. 3. (a) SABRINA prototype deployed on the roof of a building at UPC campus during an acquisition. (b) Receiver diagram of the dual channel receiver. The first channel is connected to an antenna pointed directly at the satellite to obtain a clean reference signal. The second channel receives the scattered signal. Both signals are downconverted and sampled continuously at IF. (a) SABRINA on roof of UPC. (b) SABRINA's simplified receiver diagram.

transmitter and receiver, two targets at the same bistatic range can have different phase histories. An external DEM or DTM has to be used to separate the bistatic range from the receiver and transmitter contributions and to apply the proper azimuth compression filter to each target.

\section{B. Interferometric Processing}

After the focusing step, the two resulting complex bistatic images, which are the master and slave, are combined to generate an interferogram. Fig. 4 shows the flowchart of the interferometric processing chain to generate a geocoded DEM from a pair of bistatic images. Since a DTM is available, and in order to help the phase unwrapping, it makes sense to generate a synthetic interferogram and cancel most of the interferometric fringes. Once the interferometric phase has been unwrapped, it is necessary to estimate the unknown phase offset. One option is to use a bistatic active radar calibrator (BARC) or a clear reference target with geographical coordinates that are perfectly known and mathematically calculate the phase offset. However, depending on the characteristics of the scene, it can be difficult to deploy and measure the BARC or find the proper reference. An alternative method has been developed for these situations. In both monostatic and bistatic cases, any error on

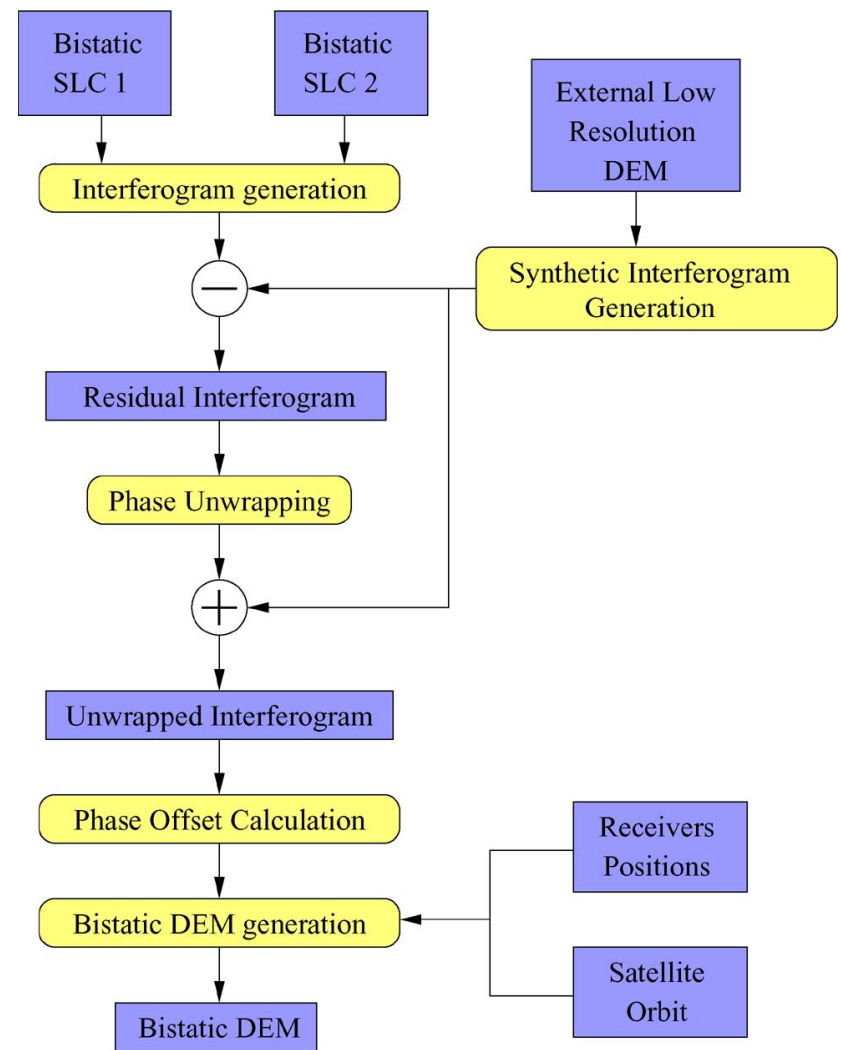

Fig. 4. Flowchart of the interferometric processing chain.

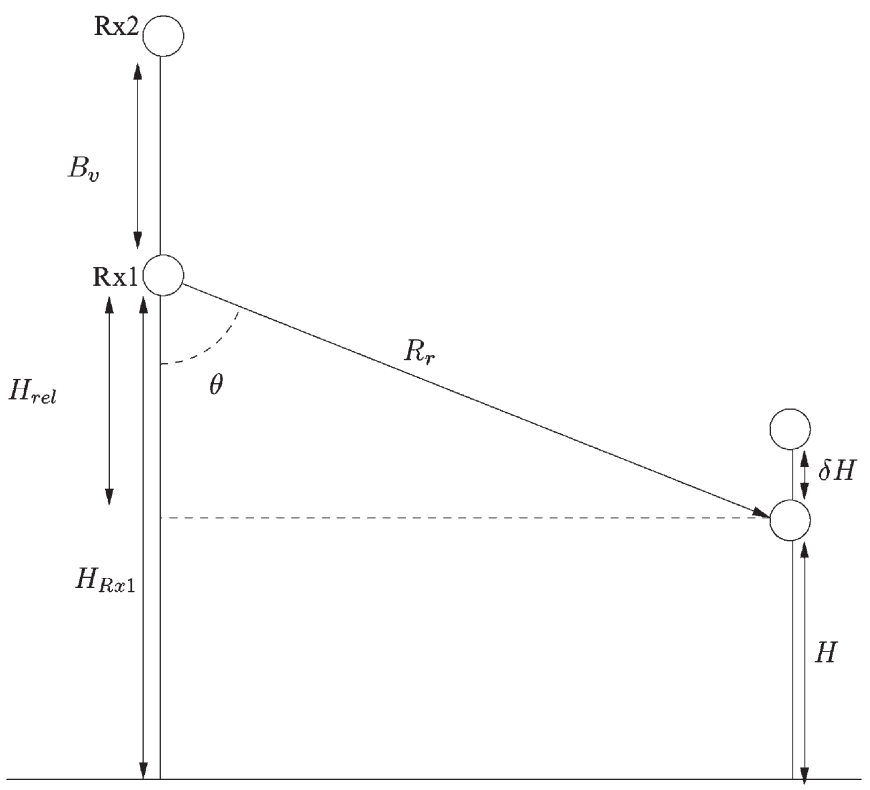

Fig. 5. Scheme used to calculate the sensitivity $\delta H / \delta \Psi$.

the phase offset would lead, as a first approximation, to a height ramp in range. In particular, for the bistatic case, the ramp is proportional to the distance to the receiver $\left(R_{r}\right)$. Similarly, the inaccuracies of the measurement of the baseline or time delays have a similar effect [26]. The sensitivity of the height as a function of the interferometric phase $(\delta H / \delta \Psi)$ can be easily derived from Fig. 5. 


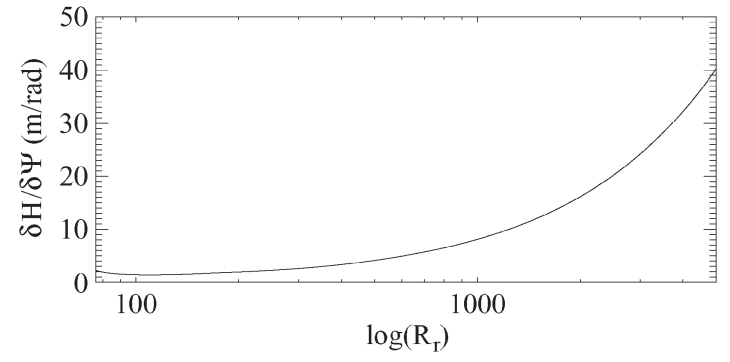

Fig. 6. Example of how the sensitivity varies along the distance to the receiver, having the receiver close to the region of interest.

For a target with a height $H$, located at a distance $R_{r}$ from the receiver, with an incidence angle $\theta$ and having a vertical baseline $B_{v}$ in reception, its interferometric phase is

$$
\Psi=\frac{2 \pi}{\lambda} \cdot B_{v} \cdot \cos (\theta) .
$$

Taking into account that

$$
\cos (\theta)=\frac{H_{\mathrm{rel}}}{R_{r}}
$$

where $H_{\mathrm{rel}}=H-H_{\mathrm{Rx} 1}$, it can be shown that

$$
\frac{\delta \Psi}{\delta H}=\frac{2 \pi}{\lambda} \cdot B_{v} \cdot \frac{\sin (\theta)^{2}}{R_{r}} .
$$

Thus, the sensitivity is given by

$$
\frac{\Delta H}{\Delta \Psi}=\frac{\lambda}{2 \pi} \cdot \frac{R_{r}}{B_{v} \cdot \sin ^{2}(\theta)} .
$$

Fig. 6 shows how the sensitivity varies with the distance to the receiver, assuming flat earth and the receiver placed at $120 \mathrm{~m}$ above sea level. This $120 \mathrm{~m}$ is the sum of the ground above sea level, which is $70 \mathrm{~m}$, and the height of the building where they are placed above the ground, which is $50 \mathrm{~m}$. The vertical baseline used in this simulation is $1.12 \mathrm{~m}$, which is the one employed in the experiments. This baseline is a good compromise between fringe frequency and hardware portability. In order to find an equivalent monostatic baseline $B_{n, \text { monostatic }}$, the bistatic and the corresponding monostatic phases can be equated

$\frac{2 \pi}{\lambda} \cdot \frac{B_{n, \text { bistatic }}}{R_{r} \cdot \sin \left(\Theta_{r, \text { bistatic }}\right)}=\frac{4 \pi}{\lambda} \cdot \frac{B_{n, \text { monostatic }}}{R_{\text {monostatic }} \cdot \sin \left(\Theta_{\text {monostatic }}\right)}$.

Taking into account that $B_{n}=B_{v} \cdot \sin (\Theta)$, assuming typical monostatic values $\left(R_{\text {monostatic }}=850 \mathrm{Km}, \Theta_{\text {monostatic }}=23^{\circ}\right)$ and a bistatic receiver range $\left(R_{r}=3 \mathrm{Km}\right)$, a vertical bistatic baseline of $1.12 \mathrm{~m}$ is equivalent to a normal monostatic baseline of $62 \mathrm{~m}$

The residual phase, which is the phase difference between the unwrapped interferogram and the synthetic one, can be related to the height of the bistatic scattering centers. Most of them belong to the visible buildings and therefore are located above the nominal height given by the starting DTM. Thus, considering a uniform distribution of building heights and that a phase offset introduces a height ramp in $R_{r}$, it makes sense

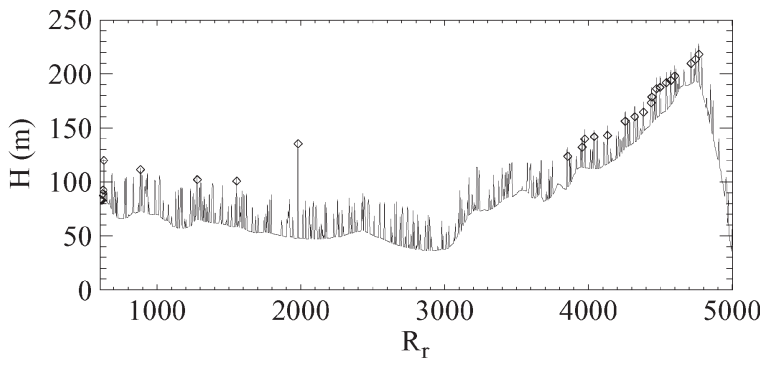

(a)

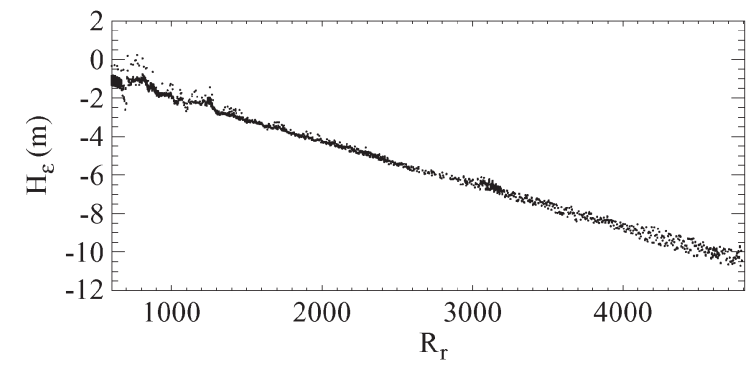

(b)

Fig. 7. (a) (Solid line) Real and (diamonds) retrieved heights of an axial cut of the simulated urban skyline. (b) Height error versus range distance for the whole simulated scene.

to find the phase offset that minimizes the absolute value of the height difference due to the residual phase.

A number of simulations have been carried out to test this phase offset estimation method with synthetic interferograms that consider the height of the buildings and shadowing. A set of urban DEMs has been generated by locating at random positions over a DTM of Barcelona obstacles chosen out of two possible uniform height distributions. The first distribution was between 0 and $40 \mathrm{~m}$, simulating the low-rise building characteristic of most of the city. The second distribution was between 40 and $100 \mathrm{~m}$, to simulate the few tall buildings present in Barcelona. Obstacles had a 300-to-1 probability of being chosen out of the first distribution. For each urban DEM, an interferogram has been computed, and random phase offsets have been added to simulate the measured data. The DTM, with no buildings, was used as a reference. Applying the sensitivity equation and minimizing the root mean squared (rms) height difference between the retrieved DEMs and the DTM, the difference between the real and obtained phase offsets always converges to approximately $15^{\circ}$. Fig. 7 shows an axial cut of one realization of the simulated DEM and the underlying DTM. The solid lines represent the real heights, and the diamonds denote the retrieved DEM for the visible points. The shadowing introduced by buildings, like the one at 2000-m range in Fig. 7(a), couples the probability of seeing a building with its height. Since the probability of being in a shadow region also increases with range, there is coupling between the measured height and distance, which is wrongly interpreted as an error due to a phase offset. The final height error due to the deviation of phase offset estimation has been analyzed for the whole simulated scene, and as it can be seen in Fig. 7(b), it is less than $12 \mathrm{~m}$ in far range. As this height error is relatively small, this method can be considered valid for the phase offset calculus in the absence of clear references in the scene. 
The fringes of the interferogram are reduced prior to the phase unwrapping using a synthetic interferogram generated with the DTM. The residual fringes are mainly due to pixels located on buildings with a height above the reference one. Using the expression of the height of ambiguity, given by

$$
H_{\mathrm{amb}}=\frac{\lambda \cdot R_{r}}{B_{v} \cdot \sin ^{2}\left(\theta_{r}\right)}
$$

and knowing approximately the maximum height of the buildings, it is possible to determine if the residual phase is likely to be wrapped. If the ambiguity height is below the maximum height of the buildings, then the phase has to be unwrapped.

Geocoding takes place using the complete interferometric phase with the phase offset corrected, the satellite's orbit, and the positions of the receiver antennas. A control point is used for orbit alignment [17].

Similar to the monostatic case, the geocoded position of each pixel can be obtained from the solution of the following system of equations:

$$
\begin{gathered}
\frac{\left(\vec{P}_{T x}-\vec{P}\right) \cdot \vec{V}_{T x}}{\|\vec{R}\|}=\lambda \cdot f_{\mathrm{DC}} \\
\left\|\vec{P}_{R x 1}-\vec{P}\right\|+\left\|\vec{P}_{T x}-\vec{P}\right\|=R_{b, 1} \\
\left\|\vec{P}_{R x 2}-\vec{P}\right\|-\left\|\vec{P}_{R x 1}-\vec{P}\right\|=-\frac{\lambda}{2 \pi} \cdot \Delta \Psi
\end{gathered}
$$

where $\vec{P}_{T x}, \vec{P}_{R x 1}, \vec{P}_{R x 2}$, and $\vec{P}$ are the Cartesian coordinates of the transmitter, the master and slave receivers, and the pixel to be georeferenced, respectively. $\vec{V}_{T x}$ is the velocity vector of the transmitter, $\lambda$ is the wavelength, $f_{\mathrm{DC}}$ is the Doppler centroid frequency, $R_{b, 1}$ is the bistatic distance for the master receiver, and $\Delta P$ si is the unwrapped interferometric phase. The first equation is the Doppler equation. Since the receiver is not moving, this equation is the same, except for a twofactor one, as in a monostatic geometry. The second equation is the bistatic range equation for the master receiver antenna that describes an ellipsoid with one focus at the master antenna and the other at the transmitter. Finally, the third equation represents a hyperbola with two foci at the receivers. This hyperboloid defines the locus of points for which the difference of distances to the master and slave antennas is constant. This difference of distances is obtained from the interferometric phase. The last equation can also be formulated as another ellipsoid for the slave antenna and its bistatic range calculated from the interferometric phase, similar of what it is done with spheres in a monostatic geometry. However, the hyperbola gives better conditioning of the nonlinear system of equations since its intersection with the ellipsoid is better defined than the intersection between two ellipsoids with a common focus at the transmitter and the other foci at each receiver.

\section{RESULTS}

In this section, the results obtained using SABRINA from two data sets with different bistatic geometries, backscattering and forward scattering, are presented and discussed. The data were acquired in Barcelona (Spain) over two different areas.

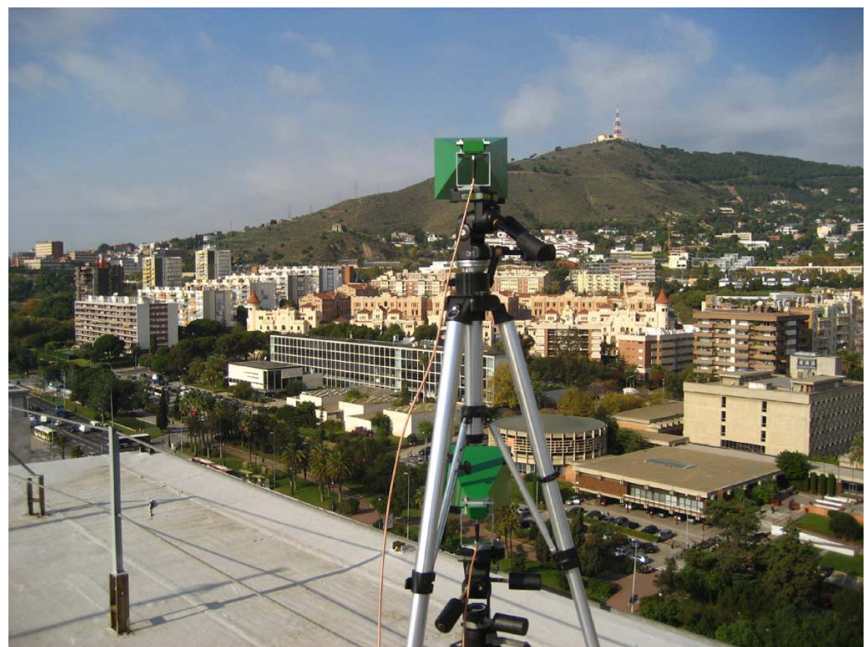

Fig. 8. Interferometric antennas and scene on the background for the backscattering geometry.

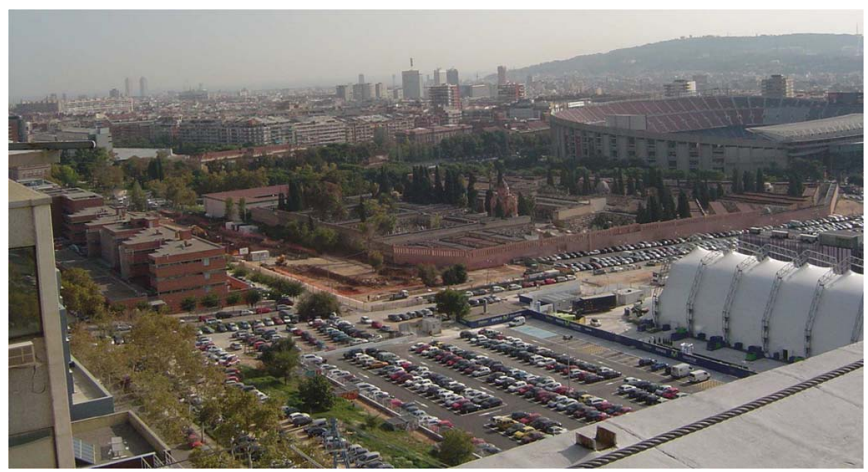

Fig. 9. Scene observed in the forward-scattering acquisition geometry.

The backscattering data were obtained using ERS-2 as the transmitter of opportunity while the forward-scattering data set using ENVISAT. In both cases, the passes were descending.

\section{A. Data Set Description}

1) Backscattering Geometry: The data acquisition was on November 9, 2007. The receivers were placed on the top of a 54-m tall building (UPC's School of Industrial Engineering), and the scene was observed under a backscattering geometry. Fig. 8 shows the experimental setup with the scene in the background: an urban area in near range and a sparsely vegetated hill on far range. The two receiver antennas were separated by a vertical baseline of $77 \mathrm{~cm}$. The maximum range was about $1 \mathrm{~km}$, which was limited by the hill in the northwest.

2) Forward-Scattering Geometry: The second acquisition took place on April 16, 2007. The system was placed in the same building, but this time, the antennas were pointing to the opposite direction, with a forward-scattering configuration. The receiver antennas were separated $112 \mathrm{~cm}$ in vertical, and the extension of the illuminated scene was larger, which is about $5 \mathrm{~km}$ in range. Most of the area is urban except a hill in the far range near the sea, called Montjuic. The scene viewed by the antennas is shown in Fig. 9. 

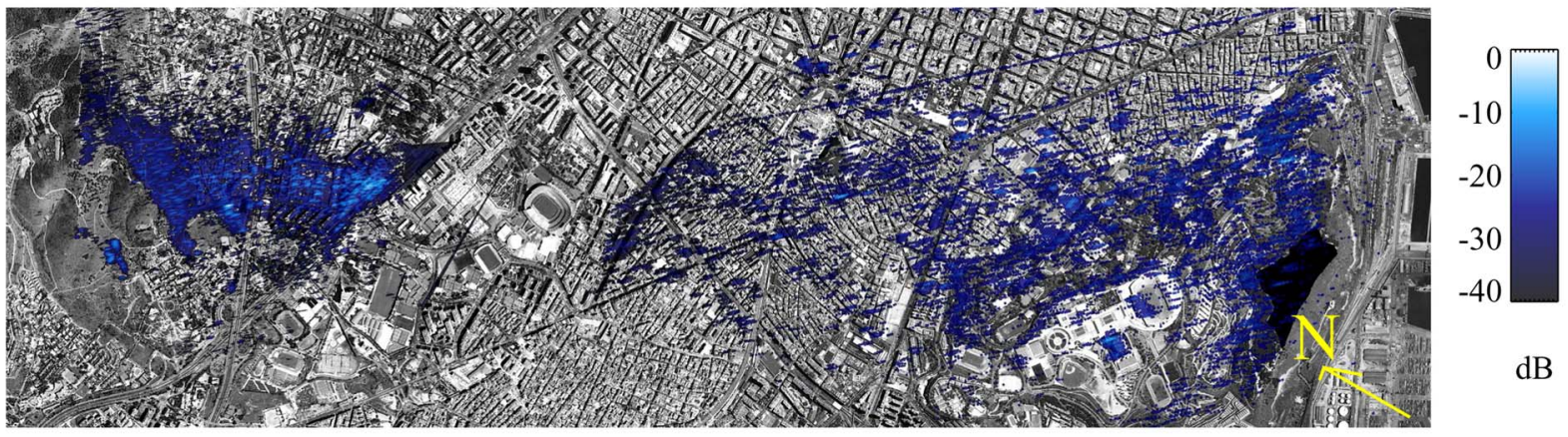

$\mathrm{dB}$

(a)
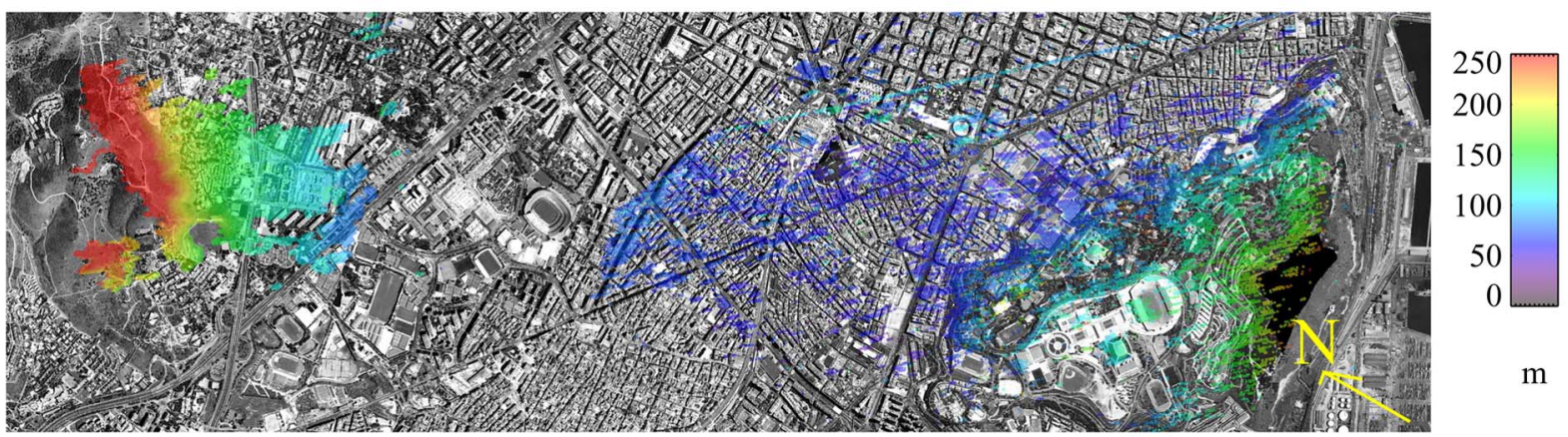

m

(b)
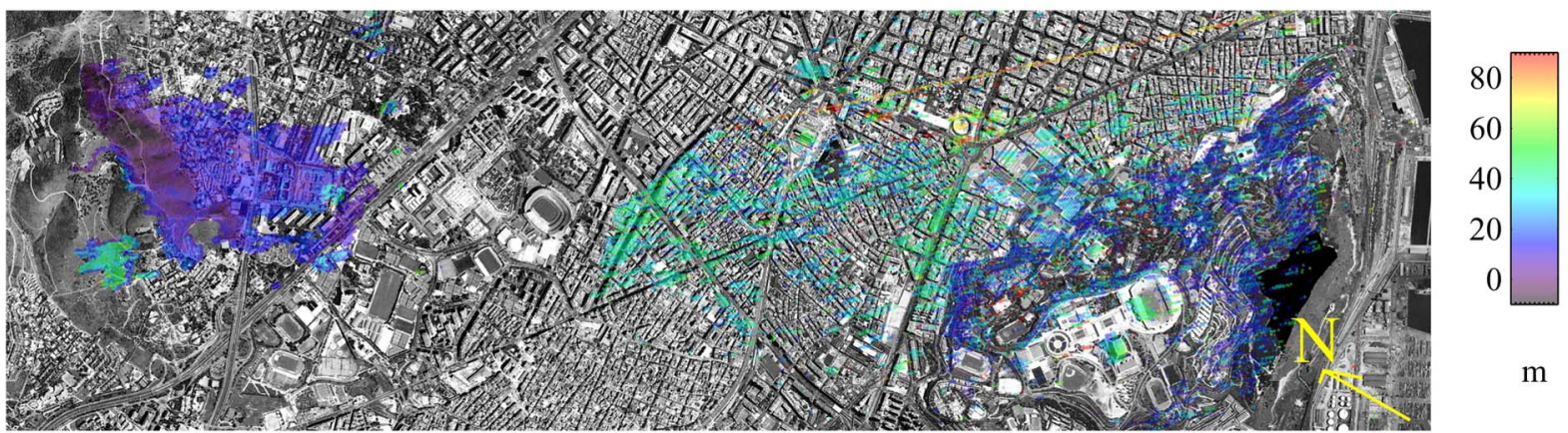

$\mathrm{m}$

(c)
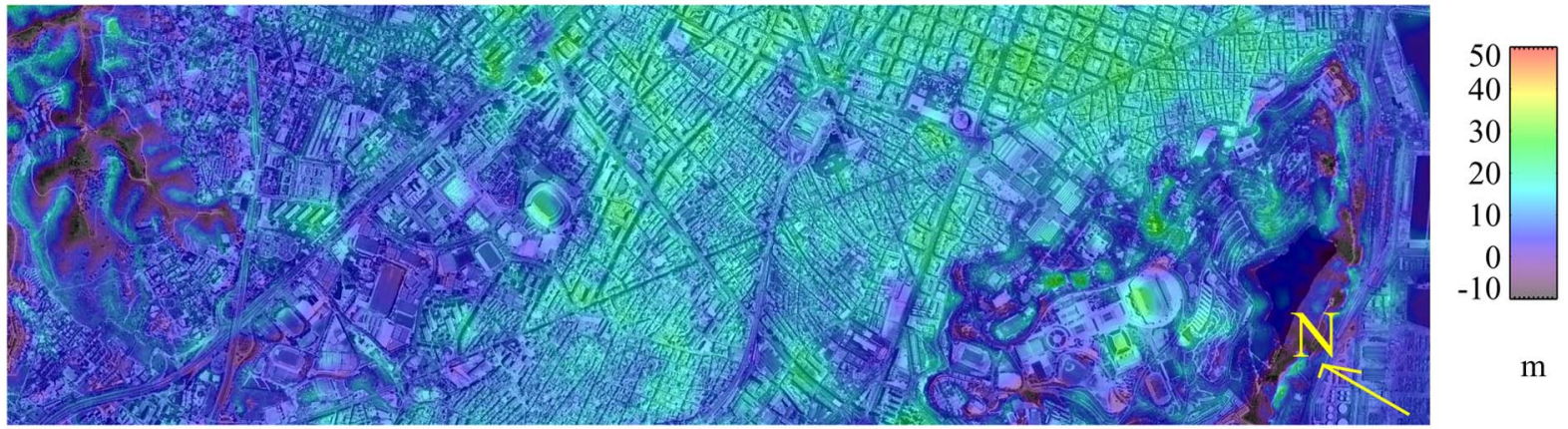

$\mathrm{m}$

(d)

Fig. 10. (a) Bistatic reflectivity of the scene. (b) Obtained DEM using the bistatic backscattering and forward-scattering interferometric data. (c) Height difference between the DEM produced using the bistatic data and the DTM. (d) Height difference between SRTM and DTM.

\section{B. Geocoded Power Images}

Fig. 10(a) shows the geocoded power of both SAR images superposed on a rotated orthophoto of the area. The backscat- tered power is located on the left side, while the forwardscattered one is on the right side of the image. The shadowing introduced by the buildings of the urban area is clearly visible 


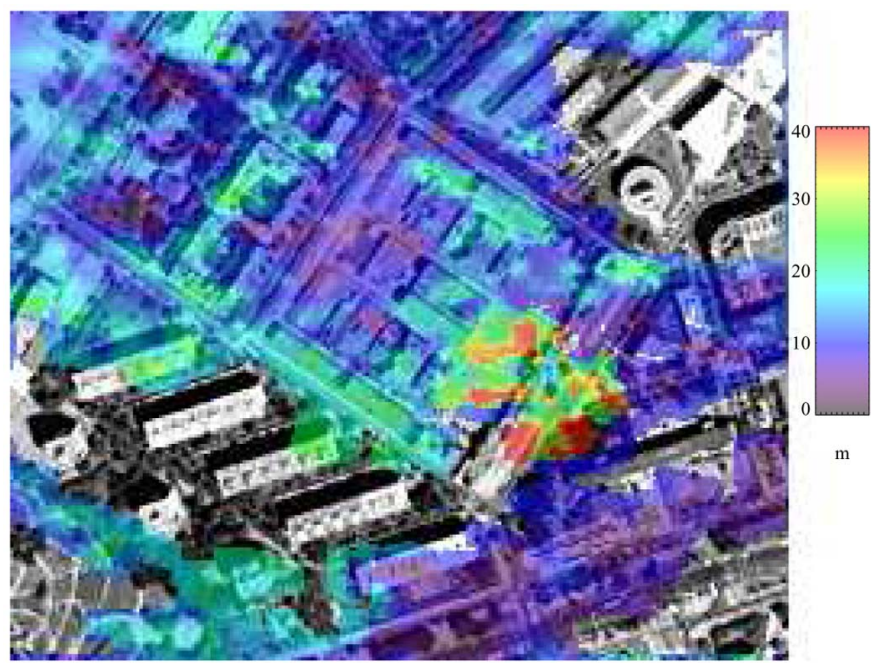

Fig. 11. Retrieved heights from a military facility.

in the forward-scattering acquisition. In this case, the doubleshadow effect inherent to bistatic radar is maximized, while in the backscattering geometry, the shaded areas are determined by the receiver as its incidence angle is larger than the incidence angle for the transmitter; see Fig. 2.

\section{DEM and Error Assessment}

The resulting DEM combining both acquisitions is shown in Fig. 10(b). The calculated DEM clearly matches the underlying topography, showing Sant Pere Martir Hill on the left and Montjuic on the right. In order to evaluate the DEM with more details and to carry out an error assessment, it has been compared with a reference DTM, which only considers the terrain but not the buildings, provided by the ICC. The difference between the bistatic DEM and the DTM is shown in Fig. 10(c). Intuitively, it is possible to observe that, in the urban area, the difference is higher and positive while, in the two bare hills, the differences are close to zero. Therefore, it can be concluded that the height differences are due to the buildings. It can be noticed that there is a straight red line at the right part of Fig. 10(c), which is due to the secondary lobes of the direct signal from the satellite. The phase of those pixels in the bistatic SAR image corresponds to the position of the receiver; thus, the residual interferogram has a wrong phase for those pixels, and consequently, an erroneous height is retrieved. The green area at the lower left part of Fig. 10(c), representing a bias of about $40 \mathrm{~m}$, corresponds to a pair of tall electricity pylons made of steel and its corresponding power lines. At the same time, the underlaying terrain is in a shadowed area.

Fig. 11 shows a detail of the retrieved height for a military facility located nearby UPC's campus. It is possible to observe the height of the walls (in green) and also the height of the two towers at the entrance.

An SRTM DEM of the scene has also been compared with the ICC's DTM. The height difference between them, which is smaller in this case, is shown in Fig. 10(d). The incidence angle of the SRTM mission was much lower than the one with the bistatic receiver. Thus, it was possible to partially see the

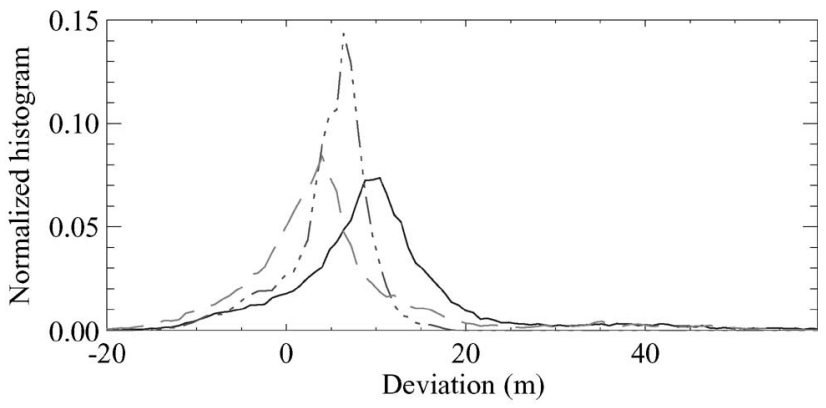

(a)

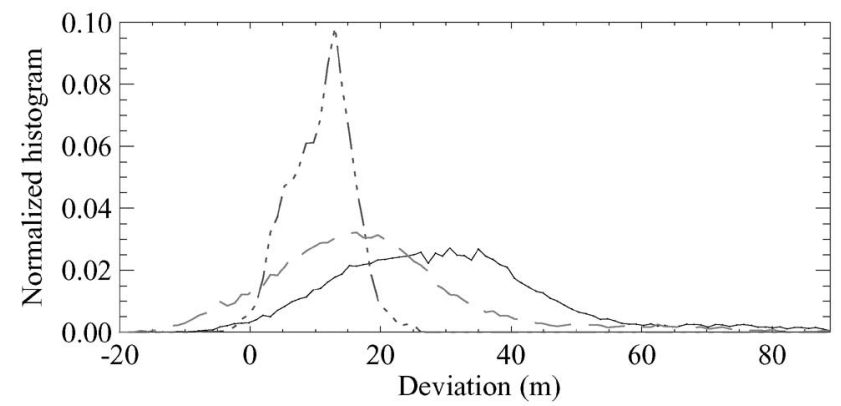

(b)

Fig. 12. Height difference normalized histograms for the (a) backscattering and (b) forward-scattering data. (Solid line) Bistatic DEM-DTM. (Big-small dashes) SRTM-DTM. (Big dashes) Bistatic DEM-SRTM.

TABLE I

BACKSCATTERING AREA Statistics

\begin{tabular}{|c|c|c|}
\hline & Mean $(\mathrm{m})$ & Std Dev $(\mathrm{m})$ \\
\hline DEM-DTM & 10.83 & 12.2 \\
\hline SRTM-DTM & 5.3 & 4.7 \\
\hline DEM-SRTM & 5.5 & 12.3 \\
\hline
\end{tabular}

streets, which have similar height than the DTM. The limited resolution of the SRTM product used, which is $90 \times 90 \mathrm{~m}$, reduces the impact of the taller buildings on the DEM as it averages all the contributions within the same resolution cell. On the contrary, the bistatic receiver mainly sees the roofs of the buildings.

To compare the height differences of the two DEMs with respect to the DTM, their normalized histograms have been calculated considering only the pixels with information in the bistatic data. The results are clearly biased by both the resolution of the product and its acquisition geometry. As commented before, the SRTM DEM has an elevation that is, in general, an average of the building heights and the terrain in edified areas while it is not biased in the rest. On the contrary, the bistatic DEM has a better resolution, and it shows mostly the height of the buildings in edified areas while it is not significantly affected by the acquisition geometry in the rest. The medium resolution DTM is not affected by any geometrical consideration. For the backscattering area, which is mostly a barely vegetated hill and low edifices, the height difference normalized histograms [Fig. 12(a)] of (solid line) bistatic DEM-DTM and (big dashes) bistatic DEM-SRTM present similar standard deviations, while the mean of the former is biased to larger values, as can be seen in Table I. The height difference normalized histogram of (bigsmall dashes) SRTM-DTM has a lower standard deviation. 


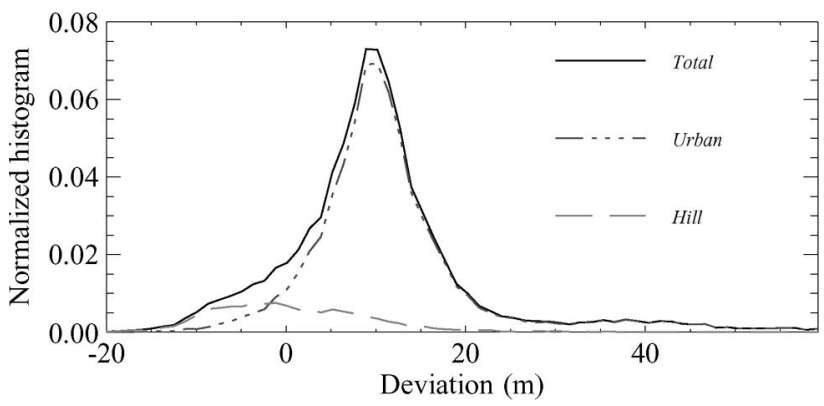

Fig. 13. Decomposition of the (solid line) DEM-DTM normalized histograms into the (big-small dashes) barely vegetated hill with no buildings and the (big dashes) urban region contributions.

TABLE II

BACKSCATTERING DEM-DTM STATISTICS DECOMPOSITION

\begin{tabular}{|c|c|c|}
\hline & Mean $(\mathrm{m})$ & Std Dev $(\mathrm{m})$ \\
\hline Total & 10.8 & 12.2 \\
\hline Urban & 12.7 & 10.5 \\
\hline Hill & 1.2 & 8.9 \\
\hline
\end{tabular}

TABLE III

ForWARD-SCATTERING AREA STATISTICS

\begin{tabular}{|c|c|c|}
\hline & Mean $(m)$ & Std Dev $(m)$ \\
\hline DEM-DTM & 33.2 & 23 \\
\hline SRTM-DTM & 11.7 & 4.9 \\
\hline DEM-SRTM & 21.4 & 22.1 \\
\hline
\end{tabular}

The barely vegetated hill with no buildings and the urban region can be easily identified for backscattering area. Thus, it is possible to decompose the normalized histograms into two contributions. Fig. 13 shows the decomposition of the DEMDTM normalized histogram; the (big-small dashes) urban, (big dashes) hill, and (solid line) total histogram are plotted. Table II shows the decomposed statistics for the DEM-DTM difference. Since the major part of the area is urban, the urban contribution is similar to the total one, presenting a bias of about $13 \mathrm{~m}$. However, the hill contribution has only a bias of $1 \mathrm{~m}$.

For the forward-scattering area, the height difference normalized histograms [Fig. 12(b)] of (solid line) bistatic DEMDTM and (big dashes) DEM-SRTM show a larger mean and a wider standard deviation than before, as presented in Table III. This is because the urban area includes a more heterogeneous distribution of buildings with different heights. The (big-small dashes) SRTM-DTM difference presents a narrower standard deviation and a lower mean than the other previous ones. This can be explained by the SRTM acquisition geometry and the way the interferometric phase offset has been calculated that bias the DEM to higher elevations.

\section{CONCLUSION}

In this paper, the application of single-pass bistatic interferometry with fixed-receiver configurations for DEM generation has been presented. The basic equations regarding image resolution and interferometric processing, including those related to geocoding, have been derived and discussed. The particularities driven by the bistatic acquisition geometry have been highlighted and compared with the classical monostatic case. The derived formulation is general, and it can be applied to the two basic acquisition geometries: forward scattering and backscattering. The development of the interferometric processing chain has also included the estimation of the unknown phase offset of the interferogram. A method not based on control points has been proposed and its limitations discussed. The phase offset can be obtained by minimizing the rms value of the height difference between the obtained DEM and a reference one. Only in urban areas, the presence of buildings combined with the high incidence angles of the fixed-receiver line of sight causes the phase offset obtained to bias the DEM to higher elevations.

The theoretical developments have been complemented by experimental results with SABRINA. Two different DEMs of the city of Barcelona have been obtained, one with a backscattering geometry and ERS as the transmitter of opportunity and the other in forward scattering and using ENVISAT. The experimental data have allowed the validation of the interferometric processing chain and have highlighted the particularities of bistatic geometries. As expected, the observation of the scene by the receiver at near grazing angles results in images that contain more scatterers corresponding to the top of the buildings than from the ground level. This phenomena has been clearly highlighted in the detailed comparisons of the retrieved bistatic DEM with a DTM, which do not include buildings, and the SRTM DEM, whose heights are an average between the elevation of the streets and those of the top of the buildings.

This paper has demonstrated the capability of bistatic systems based on a fixed receiver and orbital satellites as noncooperative transmitters to produce DEMs. The DEMs are not the final application we are foreseeing but the first logical step toward the development of a monitoring tool of subsidence phenomena. The next steps are oriented to the obtention of repeat-pass interferograms, the acquisition of an interferometric data set in an area affected by urban subsidence to which apply advanced DInSAR techniques and the development of a truly multistatic system with a larger number of receivers deployed.

\section{ACKNOWLEDGMENT}

DEM was provided by the NASA/DLR SRTM mission and the DTM by the ICC.

\section{REFERENCES}

[1] D. Massonet and T. Rabaute, "Topographic mapping from interferometer synthetic aperture radar observations," J. Geophys. Res., vol. 91, no. B5, pp. 4993-4999, 1986.

[2] C. Prati, F. Rocca, A. M. Guarnieri, and E. Damonti, "Seismic migration for SAR focusing: Interferometrical applications," IEEE Trans. Geosci. Remote Sens., vol. 28, no. 4, pp. 627-640, Jul. 1990.

[3] D. Massonet and T. Rabaute, "Radar interferometry: Limits and potential," IEEE Trans. Geosci. Remote Sens., vol. 31, no. 2, pp. 455-464, Mar. 1993.

[4] R. F. Hanssen, Radar Interferometry. Data Interpretation and Error Analysis. Norwell, MA: Kluwer, 2001, ch. 4.

[5] A. Ferretti, C. Prati, and F. Rocca, "Permanent scatterers in SAR interferometry," IEEE Trans. Geosci. Remote Sens., vol. 39, no. 1, pp. 8-20, Jan. 2001. 
[6] P. Berardino, G. Fornaro, R. Lanari, and E. Sansosti, "A new algorithm for surface deformation monitoring based on small baseline differential SAR interferograms," IEEE Trans. Geosci. Remote Sens., vol. 40, no. 11, pp. 2375-2383, Nov. 2002.

[7] O. Mora, J. J. Mallorqui, and A. Broquetas, "Linear and nonlinear terrain deformation maps from a reduced set of interferometric SAR images," IEEE Trans. Geosci. Remote Sens., vol. 41, no. 10, pp. 2243-2253, Oct. 2003.

[8] S. R. Cloude and E. Pottier, "A review of target decomposition theorems in radar polarimetry," IEEE Trans. Geosci. Remote Sens., vol. 34, no. 2, pp. 498-518, Mar. 1996.

[9] S. R. Cloude and K. P. Papathanassiou, "Polarimetric SAR interferometry," IEEE Trans. Geosci. Remote Sens., vol. 36, no. 5, pp. 1551-1565, Sep. 1998.

[10] S. R. Cloude, "On the status of bistatic polarimetry theory," in Proc. Int. Geosci. Remote Sens. Symp., 2005, vol. 3, pp. 2003-2006.

[11] A. Moreira, G. Krieger, I. Hajnsek, D. Hounam, M. Werner, S. Riegger, and E. Settelmeyer, "TanDEM-X: A TerraSAR-X add-on satellite for single-pass SAR interferometry," in Proc. Int. Geosci. Remote Sens. Symp., 2004, vol. 2, pp. 1000-1003.

[12] I. Walterscheid, J. H. Ender, A. R. Brenner, and O. Loffeld, "Bistatic SAR processing and experiments," IEEE Trans. Geosci. Remote Sens., vol. 44, no. 10, pp. 2710-2717, Oct. 2006.

[13] J. Klare, I. Walterscheid, A. R. Brenner, and J. H. G Ender, "Evaluation and optimisation of configurations of a hybrid bistatic SAR experiment between TerraSAR-X and PAMIR," in Proc. Int. Geosci. Remote Sens. Symp., 2006, pp. 1208-1211.

[14] J. Klare, J. H. G Ender, O. Loffeld, A. R. Brenner, and I. Walterscheid, "Bistatic exploration using spaceborne and airborne SAR sensors-A close collaboration between FGAN, ZESS, and FOMAAS," in Proc. Int. Geosci. Remote Sens. Symp., 2006, pp. 1828-1831.

[15] M. Zink, H. Fiedler, I. Hajnsek, G. Krieger, A. Moreira, and M. Werner, "The tanDEM-X mission concept," in Proc. Int. Geosci. Remote Sens. Symp., 2006, pp. 1938-1941.

[16] G. Krieger, A. Moreira, H. Fiedler, I. Hajnsek, M. Werner, and M. Younis, "TanDEM-X: A satellite formation for high-resolution SAR interferometry," IEEE Trans. Geosci. Remote Sens., vol. 45, no. 11, pp. 3317-3341, Jul.-Nov. 2007.

[17] P. Lopez-Dekker, J. J. Mallorqui, P. Serra-Morales, and J. Sanz-Marcos, "Phase synchronization and Doppler centroid estimation in fixed receiver bistatic SAR systems," IEEE Trans. Geosci. Remote Sens., vol. 46, no. 11, pp. 3459-3471, Nov. 2008.

[18] J. Sanz-Marcos, P. Prats, J. Mallorqui, and A. Aguasca, "A subaperture range-Doppler processor for bistatic-fixed-receiver SAR," in Proc. EUSAR, 2006. CD-ROM

[19] J. Sanz-Marcos, P. Lopez-Dekker, J. J. Mallorqui, A. Aguasca, and P. Prats, "SABRINA: A SAR bistatic receiver for interferometric applications," IEEE Geosci. Remote Sens. Lett., vol. 4, no. 2, pp. 307-311, Apr. 2007.

[20] J. Sanz-Marcos, J. Mallorqui, and T. Broquetas, "Bistatic parasitic SAR processor evaluation," in Proc. Int. Geosci. Remote Sens. Symp., 2004, pp. 3666-3669.

[21] J. Sanz-Marcos, P. Prats, and J. Mallorqui, "Bistatic fixed receiver parasitic SAR processor based on the back-propagation algorithm," in Proc. Int. Geosci. Remote Sens. Symp., 2005, pp. 1056-1059.

[22] J. Sanz-Marcos, J. Mallorqui, A. Aguasca, and P. Prats, "First ENVISAT and ERS-2 parasitic bistatic fixed receiver SAR images processed with the subaperture range-Doppler algorithm," in Proc. Int. Geosci. Remote Sens. Symp., 2006, pp. 1840-1843.

[23] F. Daout, V. Giroux, and H. Cantalloube, "An omega- $k$ algorithm for SAR bistatic systems," in Proc. Int. Geosci. Remote Sens. Symp., 2005, pp. 1060-1063.

[24] O. Loffeld, H. Nies, V. Peters, and S. Knedlik, "Models and useful relations for bistatic SAR processing," in Proc. Int. Geosci. Remote Sens. Symp., 2003, pp. 1442-1445.

[25] A. Monti Guarnieri and F. Rocca, "Reduction to monostatic focusing of bistatic or motion uncompensated SAR surveys," Proc. Inst. Elect. Eng.-Radar, Sonar Navig., vol. 153, no. 3, pp. 199-207, Jun. 2006.

[26] J. J. Mallorqui, M. Bara, and A. Broquetas, "Calibration requirements for airborne SAR interferometry,” Proc. SPIE—Int. Soc. Opt. Eng., vol. 4173, pp. 267-278, Sep. 2000.

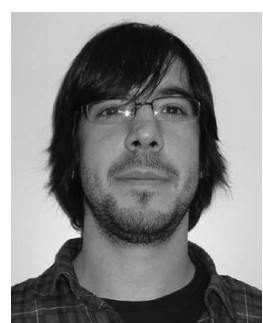

Sergi Duque (S'08) was born in Barcelona, Spain, in 1981. He received the Ingeniero degree in telecommunication from the Universitat Politècnica de Catalunya, Barcelona, in 2005, where he is currently working toward the Ph.D. degree.

In 2008, he was with the University of Michigan, Ann Arbor, for a period of six months, working on 3-D bistatic SAR image focusing. His main research topics are bistatic SAR interferometry (InSAR), differential InSAR, and bistatic tomography.

Mr. Duque was awarded with the 2nd Prize of the X Premio Rosina Ribalta given by the EPSON Foundation to the Best National Thesis Proposals in the field of information and communication technologies in 2008 .

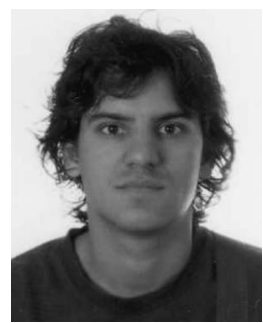

Paco (Francisco) López-Dekker (S'98-M'03) was born in Nijmegen, The Netherlands, in 1972 . He received the Ingeniero degree in telecommunication engineering from the Universitat Politècnica de Catalunya (UPC), Barcelona, Spain, in 1997, the M.S. degree in electrical and computer engineering from the University of California, Irvine, in 1998, under the Balsells Fellowships, and the Ph.D. degree from the University of Massachusetts, Amherst, in 2003, for his research on clear-air imaging radar systems to study the atmospheric boundary layer.

In 1999, he was with the Microwave Remote Sensing Laboratory, University of Massachusetts. In 2003, he returned to Barcelona to work for Starlab, which is a privately held company, to work on the development of Global Navigation Satellite System Reflection sensors. In 2004, he joined the Telecommunications and Systems Engineering Department, Universitat Autonoma de Barcelona, Barcelona, as a Visiting Professor. In 2005, he was awarded a five-year Ramon y Cajal Grant to conduct research on bistatic SAR at the Remote Sensing Laboratory, UPC, where he joined in March 2006. At the university, he has taught courses on signals and systems, signal processing, communications systems and radiation, and guided waves. Since November 2009, he has been with the Microwaves and Radar Institute, German Aerospace Center (DLR), Oberpfaffenhofen, Germany. His current research is focused on the study of future SAR missions and novel mission concepts.

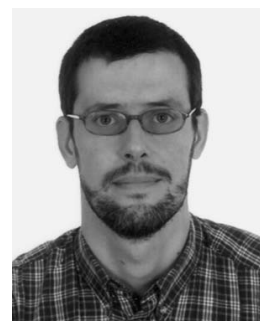

Jordi J. Mallorqui (S'93-M'96) was born in Tarragona, Spain, in 1966. He received the Ingeniero degree in telecommunications engineering and the Doctor Ingeniero degree in telecommunications engineering for his research on microwave tomography for biomedical applications from the Department of Signal Theory and Communications, Universitat Politècnica de Catalunya (UPC), Barcelona, Spain, in 1990 and 1995, respectively.

Since 1993, he has been teaching at the School of Telecommunications Engineering of Barcelona, UPC, first as an Assistant Professor and then, since 1997, as an Associate Professor. His teaching activity involves microwaves, radio navigation systems, and remote sensing. He spent a sabbatical year with the Jet Propulsion Laboratory, Pasadena, CA, in 1999, working on interferometric airborne synthetic aperture radar (SAR) calibration algorithms. He is currently working on the application of SAR interferometry to terrain-deformation monitoring with orbital, airborne, and ground data; vessel detection and classification from SAR images; and 3-D electromagnetic (EM) simulation of SAR systems. He is also collaborating in the design and construction of a ground-based SAR interferometer for landslide control. Finally, he is currently developing the hardware and software of a bistatic opportunistic SAR for interferometric applications using ERS, ENVISAT, and TerraSAR-X as sensors of opportunity. He has published more than 80 papers on microwave tomography, EM numerical simulation, and SAR processing, interferometry, and differential interferometry in refereed journals and international symposia. 